Classification

Physics abstracts

$16.35-23.40$

\section{SUR UN DISPOSITIF SIMPLE DESTINÉ A LA DIFFRACTOMÉTRIE X DE POUDRE A BASSE TEMPÉRATURE}

\author{
L. TRUT, J. SENEGAS et J. GALY
}

Service de Chimie Minérale Structurale de l'Université de Bordeaux I, associé au CNRS, 351, cours de la libération, 33405 Talence, France

(Reçu le 11 octobre 1972, revisé le 30 octobre 1972)

Résumé. - L'appareil décrit permet l'obtention de diffractogrammes de rayons $\mathbf{X}$ à des températures régulées à $\pm 0,5^{\circ} \mathrm{K}$ entre $65^{\circ} \mathrm{K}$ et $320^{\circ} \mathrm{K}$.

Abstract. - The apparatus described gives X-rays powder diagram at temperatures precise to $\pm 0.5^{\circ} \mathrm{K}$ in the range of $65^{\circ} \mathrm{K}$ to $320^{\circ} \mathrm{K}$.

1. Introduction. - L'étude des propriétés physiques de matériaux en liaison avec leur structure nécessite la connaissance de leurs données cristallographiques dans un large domaine de température.

La diffraction $X$ est particulièrement utile lorsque se produit une transformation allotropique dans le domaine considéré.

Nous avons ainsi été amenés à mettre au point un dispositif de diffractométrie $X$ de poudre à basse température fonctionnant entre la température ambiante et celle de l'azote liquide.

2. Description de l'installation. - 2.1 ENSEMBLE DE DIFFRACTOMÉTRIE. - Le goniomètre horizontal est de type Philips PW 1380.

2.2 CRyostat et montage SUR Le Goniomètre. Le schéma du cryostat modifié est donné à la figure 1 .

Le cryostat est fixé sur l'axe du goniomètre grâce à un cylindre en lucoflex. Il comprend deux parties :

- l'intérieur constitué du porte-échantillon hémicylindrique dans lequel sont incorporés la sonde de mesure de température et un capillaire destiné à la circulation de l'azote liquide;

- l'extérieur comportant au niveau du porteéchantillon une fenêtre en mylar. Celle-ci collée sur l'acier à l'araldite permet aux rayons $X$ de pénétrer tout en assurant l'isolement de l'échantillon.

Enfin une vanne permet de faire le vide à l'intérieur $\mathrm{du}$ cryostat.

2.3 Mesure et RÉGULATION DE LA TEMPÉRATURE. La sonde délivre un signal électrique, mesuré sur un millivoltmètre AOIP. La variation est linéaire avec la température. La sensibilité est de l'ordre de $0,1^{\circ} \mathrm{C}$.

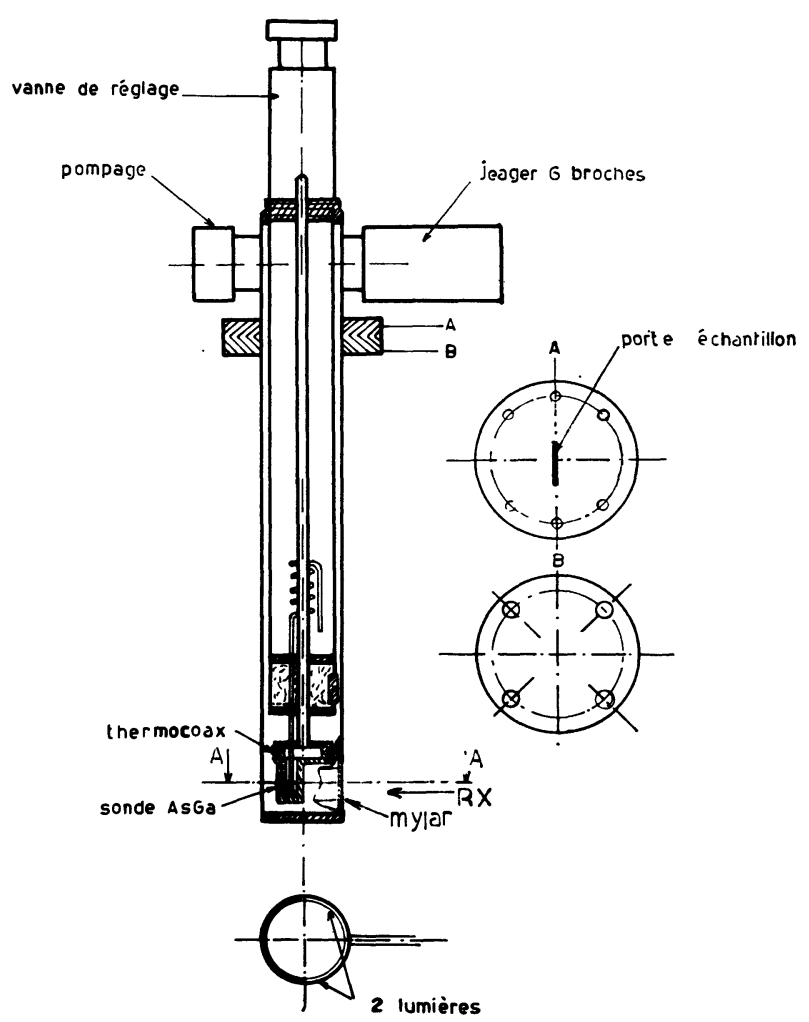

FIG. 1. - Schéma d'ensemble de la chambre de diffraction avec son cryostat.

La position de la sonde, en contact direct avec le porte-échantillon, nous assure d'un très faible écart entre la température lue et celle de l'échantillon de poudre.

Les modifications de températures et leur régulation s'obtiennent par effet Joule à l'aide d'un enroulement thermoax. 
Les limites de température d'utilisation sont, d'une part, celle de l'azote liquide $\left(77^{\circ} \mathrm{K}\right)$ que l'on peut éventuellement diminuer par pompage jusque vers $65^{\circ} \mathrm{K}$ et d'autre part $320^{\circ} \mathrm{K}$, limite maximale de température pour éviter toute détérioration de la sonde à l'arséniure de gallium.

3. Etalonnage. - La sonde était étalonnée simplement pour deux températures: $77^{\circ} \mathrm{K}$ et $293^{\circ} \mathrm{K}$. Nous avons vérifié la linéarité du signal de tension avec la température entre ces deux points. Pour ce faire nous avons eu recours à l'étude dilatométrique de deux échantillons, l'un en or et l'autre en chlorure de potassium (Fig. 2).

\begin{tabular}{|c|c|c|}
\hline & $\begin{array}{l}\alpha \cdot 10^{6} \cdot \mathrm{d}^{-1} \\
\text { Ce travail }\end{array}$ & $\begin{array}{l}\alpha .10^{6} . \mathrm{d}^{-1} \\
\text { Littérature }\end{array}$ \\
\hline $\mathrm{Au}$ & 12,9 & $11,7<\alpha .10^{6}<13,1$ \\
\hline $\mathrm{KCl}$ & 31,5 & $29,7<\alpha .10^{6}<33,7$ \\
\hline
\end{tabular}

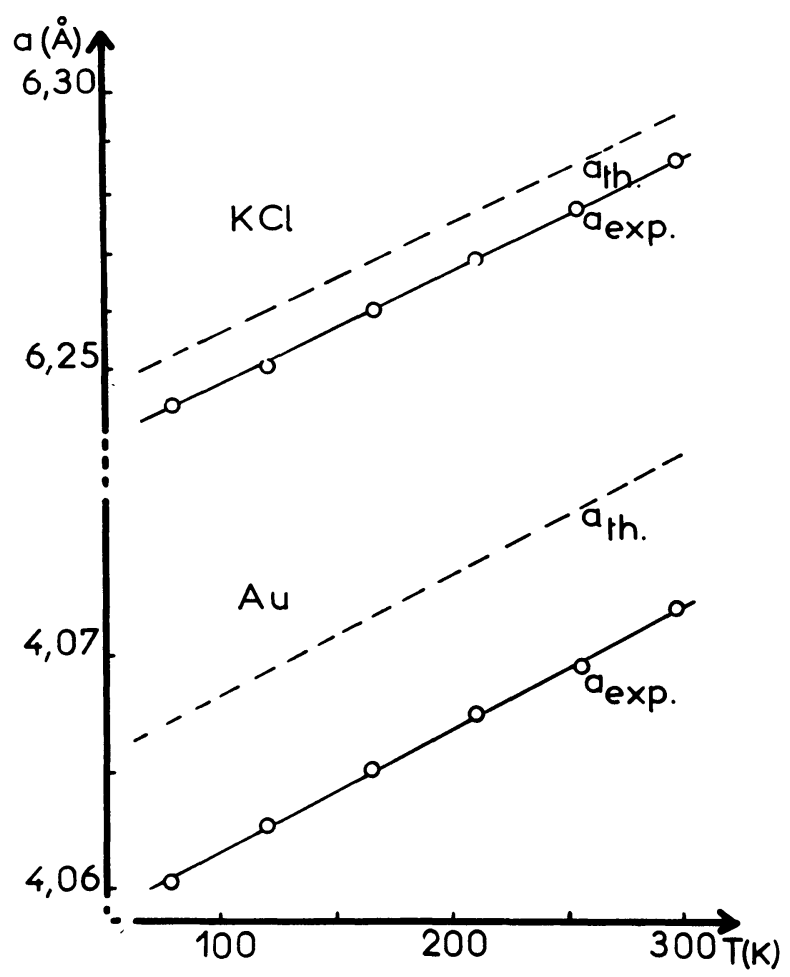

Fig. 2. - Evolution du paramètre de l'or et du chlorure de potassium avec la température.
4. Exemple d'application. - Détermination des transitions de phases du titanate de baryum et du sesquioxyde de vanadium : $\mathrm{BaTiO}_{3}$ et $\mathrm{V}_{2} \mathrm{O}_{3}$.

4.1 Titanate De baryum. - Le titanate de baryum présente cinq transitions en fonction de la température (3).

Nous nous sommes attachés à suivre son évolution en dessous de la température ambiante en observant une raie caractéristique correspondant aux indexations suivantes :

$$
\begin{aligned}
& \text { rhomboédrique : (200) } \\
& \text { orthorhombique (pseudoquadratique) } \\
& \text { quadratique }\left\{\begin{array}{l}
(002) \\
(200)
\end{array}\right.
\end{aligned}
$$

L'évolution du paramètre $a$ de la phase rhomboédrique et $a$ et $c$ des phases orthorhombiques et quadratiques (Fig. 3) pour des températures croissant de $77^{\circ} \mathrm{K}$ à $300 \% \mathrm{~K}$ montre que ces transitions sont brutales et surviennent à des températures bien définies (rhomboédrique $\rightarrow$ orthorhombique : $190^{\circ} \mathrm{K}$, orthorhombique $\rightarrow$ quadratique : $270^{\circ} \mathrm{K}$ ).

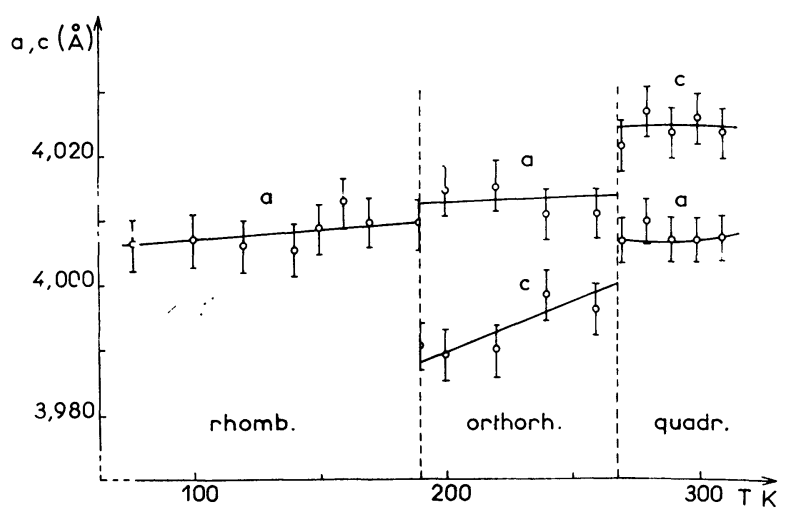

FIG. 3. - Variation des paramètres $a$ et $c$ du titanate de baryum avec la température.

4.2 SesquioXYde $\mathrm{V}_{2} \mathrm{O}_{3}$. - Le sesquioxyde de vanadium subit une transition métal $\leftrightharpoons$ isolant réversible vers $170^{\circ} \mathrm{K}$. La phase haute température de symétrie rhomboédrique possède la structure corindon. A basse température $\mathrm{V}_{2} \mathrm{O}_{3}$ est un isolant, la structure corindon se déforme légèrement, la symétrie devient monoclinique.

Nous avons observé cette transformation qui se produit de manière assez brutale.

\section{Bibliographie}

[1] Hebert H., Z. Phys. 47 (1928) 719.

[2] Fizeau R., C. R. Acad. Sci. Paris 64 (1867) 314

[3] Kay H. F. et Voosden P., Phil. Mag. 40 (1949) 1019. 